\title{
Central Catadioptric Visual Servoing From 3D Straight Lines
}

\author{
Y. Mezouar \\ H. Haj Abdelkader \\ LASMEA \\ 24 avenue des Landais \\ 63177 AUBIERE-FRANCE \\ mezouar,hadj,martinet@lasmea.univ-bpclermont.fr
}

\author{
F. Chaumette \\ IRISA \\ Campus de Beaulieu \\ RENNES-FRANCE \\ chaumette@irisa.fr
}

\begin{abstract}
In this paper we consider the problem of controlling a robotic system using the projection of $3 D$ lines in the image plane of central catadioptric systems. Most of the effort in visual servoing are devoted to points, only few works have investigated the use of lines in visual servoing with traditional cameras and none has explored the case of omnidirectional cameras. First a generic central catadioptric interaction matrix for the projection of 3D straight lines is derived from the projection model of an entire class of camera. Then an image-based control law is designed and validated through simulation results.
\end{abstract}

\section{INTRODUCTION}

Vision-based servoing schemes are flexible and effective methods to control robot motions from cameras observations [13]. They are traditionally classified into three groups, namely position-based, image-based and hybridbased control [11], [13], [15]. These three schemes make assumptions on the link between the initial, current and desired images since they require correspondences between the visual features extracted from the initial image with those obtained from the desired one. These features are then tracked during the camera (and/or the object) motion. If these steps fail the visually based robotic task can not be achieved [8]. Typical cases of failure arise when matching joint images features is impossible (for example when no joint features belongs to initial and desired images) or when some parts of the visual features get out of the field of view during the servoing. Some methods have been investigated to resolve this deficiency based on path planning [16], switching control [9], zoom adjustment [18], geometrical and topological considerations [10], [20]. However, such strategies are sometimes delicate to adapt to generic setup.

Conventional cameras suffer thus from restricted field of view. Many applications in vision-based robotics, such as mobile robot localisation [6] and navigation [22], can benefit from panoramic field of view provided by omnidirectional cameras. In the literature, there have been several methods proposed for increasing the field of view of cameras systems [5]. One effective way is to combine mirrors with conventional imaging system. The obtained sensors are referred as catadioptric imaging systems. The resulting imaging systems have been termed central catadioptric when a single projection center describes the world-image mapping. From a theoretical and practical view point, a single center of projection is a desirable property for an imaging system [2]. Baker and Nayar in [2] derive the entire class of catadioptric systems with a single viewpoint. Clearly, visual servoing applications can also benefit from such sensors since they naturally overcome the visibility constraint. Vision-based control of robotic arms, single mobile robot or formation of mobile robots appear thus in the literature with omnidirectional cameras (refer for example to [4], [7], [17], [21]).

The interaction matrix plays a central role to design vision-based control law. It links the variations of image observations to the camera velocity. The analytical form of the interaction matrix is available for some image features (points, circles, lines, $\cdots$ ) in the case of conventional cameras [11]. Barreto et al. in [4] studied the central catadioptric interaction matrix for a set of image points. This paper is mainly concerned with the use of projected lines extracted from central catadioptric images as input of a visual servoing control loop. When dealing with real environments (indoor or urban) or industrial workpiece, lines features are natural choices. Nevertheless, most of the effort in visual servoing are devoted to points [13], only few works have investigated the use of lines in visual servoing with traditional cameras (refer for example to [1], [11], [14]) and none has explored the case of omnidirectional cameras. This paper is concerned with this last issue. We derive a generic analytical form of the central catadioptric interaction matrix for the image of 3D straight lines which can be exploited to design control laws for positioning task of a six degrees of freedom manipulator or for trajectory following task for a mobile robot.

The remainder of this paper is organized as follows. In Section II, following the description of the central catadioptric camera model, lines projection in the image plane is studied. This is achieved using the unifying theory for central panoramic systems introduced in [12]. In Section III the classical image-based control law we have used, is briefly presented. We derive then a generic analytical form of the interaction matrix for projected lines (conics) and finally, we focus on the case of cameras combining a parabolic mirror and an orthographic camera. In Section IV, simulated results are presented.

\section{Central CAtadioptric PRojection OF Lines}

In this section, we describe the projection model for central catadioptric cameras and then we focus on 3D lines features. 


\begin{tabular}{|l|c|c|c|}
\hline camera & Mirror surface & $\xi$ & $\varphi$ \\
\hline Parabolic & $z=\frac{x^{2}+y^{2}}{2 a_{p}}-\frac{a_{p}}{2}$ & 1 & $1+2 p$ \\
\hline Hyperbolic & $\frac{\left(z+\frac{d}{2}\right)^{2}}{a_{h}^{2}}-\frac{x^{2}+y^{2}}{b_{h}^{2}}=1$ & $\frac{d}{\sqrt{d^{2}+4 p^{2}}}$ & $\frac{d+2 p}{\sqrt{d^{2}+4 p^{2}}}$ \\
\hline Elliptical & $\frac{\left(z+\frac{d}{2}\right)^{2}}{a_{e}^{2}}+\frac{x^{2}+y^{2}}{b_{e}^{2}}=1$ & $\frac{d}{\sqrt{d^{2}+4 p^{2}}}$ & $\frac{d-2 p}{\sqrt{d^{2}+4 p^{2}}}$ \\
\hline Planar & $z=\frac{d}{2}$ & 0 & 1 \\
\hline conventional & none & 0 & 1 \\
\hline
\end{tabular}

TABLE I

CENTRAL CATADIOPTRIC CAMERAS DESCRIPTION: $a_{p}, a_{h}, b_{h}, a_{e}, b_{e}$ depend only of the mirror intrinsic parameters $d$ and $p$

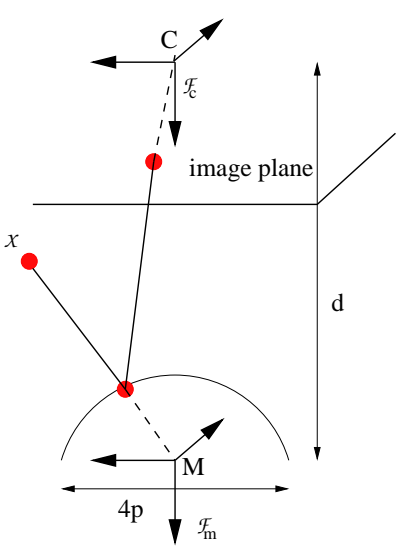

Fig. 1. Generic camera model

\section{A. Camera model}

As noted previously, a single center of projection is a desirable property for an imaging system. A single center implies that all lines passing through a $3 \mathrm{D}$ point and its projection in the image plane pass through a single point in 3D space. Conventional perspective cameras are single view point sensors. As shown in [2], a central catadioptric system can be built by combining an hyperbolic, elliptical or planar mirror with a perspective camera and a parabolic mirror with an orthographic camera. To simplify notations conventional perspective cameras will be embedded in the set of central catadioptric cameras. In [12], a unifying theory for central panoramic systems is presented. According to this generic model, all central panoramic cameras can be modeled by a central projection onto a sphere followed by a central projection onto the image plane (see Fig. 1). This generic model can be parametrized by the couple $(\xi, \varphi)$ (see Tab.I and refer to [4]). Let $\mathcal{F}_{c}$ and $\mathcal{F}_{m}$ be the frames attached to the conventional camera and to the mirror respectively. In the sequel, we suppose that $\mathcal{F}_{c}$ and $\mathcal{F}_{m}$ are related by a translation along the $\mathrm{Z}$-axis. The centers $C$ and $M$ of $\mathcal{F}_{c}$ and $\mathcal{F}_{m}$ will be termed optical center and principal projection center respectively. Let $\mathcal{X}$ be a $3 \mathrm{D}$ point with coordinates $\mathbf{X}=\left[\begin{array}{lll}X & Y & Z\end{array}\right]^{T}$ with respect to $\mathcal{F}_{m}$. According to the generic projection model [12], $\mathcal{X}$ is projected in the image plane to a point $\mathbf{x}=\left[\begin{array}{lll}x & y & 1\end{array}\right]^{T}$ with:

$$
\mathbf{x}=\mathbf{K M f}(\mathbf{X})
$$

where $\mathbf{K}$ denotes the triangular calibration matrix of the conventional camera, and:

$$
\mathbf{M}=\left[\begin{array}{ccc}
\varphi-\xi & 0 & 0 \\
0 & \varphi-\xi & 0 \\
0 & 0 & 1
\end{array}\right] \quad \mathbf{f}(\mathbf{X})=\left(\begin{array}{c}
\frac{X}{Z+\xi \sqrt{X^{2}+Y^{2}+Z^{2}}} \\
\frac{Y}{Z+\xi \sqrt{X^{2}+Y^{2}+Z^{2}}} \\
1
\end{array}\right)
$$

In the sequel, we will assume without loss of generality that the matrices $\mathbf{K}$ and $\mathbf{M}$ are equal to the identity matrix, the mapping function describing central catadioptric projection is then given by $\mathbf{x}=\mathbf{f}(\mathbf{X})$

\section{B. Projection of Lines}

In order to model lines projection in the image of a central imaging system, we use the Plücker coordinates of lines (refer to Fig. 2). Let $\mathcal{P}$ be a 3D point and $\mathbf{u}=$ $\left(u_{x}, u_{y}, u_{z}\right)^{T}$ a $(3 \times 1)$ vector expressed in the mirror frame and $\mathcal{L}$ the 3 -D line they define. Define $\mathbf{n}=\frac{\overrightarrow{M \mathcal{P}}_{\times \mathbf{u}}}{\left\|\overrightarrow{M \mathcal{P}}_{\times \mathbf{u}}\right\|}=$ $\left(n_{x}, n_{y}, n_{z}\right)^{T}$ and remark that this vector is independent of the point we choose on the line. Thus the Euclidean Plücker coordinates are defined as $\mathcal{L}:\left(\begin{array}{ll}\mathbf{n}^{T} & \mathbf{u}^{T}\end{array}\right)^{T}$ with $\|\mathbf{n}\|=1$ and $\mathbf{n}^{T} \mathbf{u}=0$. The $\mathbf{n}$-vector is orthogonal to the interpretation plane $\Pi$ defined by the line and the principal projection center:

$$
\mathbf{X}=[X, Y, Z]^{T} \in \Pi \Longleftrightarrow n_{x} X+n_{y} Y+n_{z} Z=0
$$

Let $\mathcal{S}$ be the intersection between the interpretation plane and the mirror surface. $\mathcal{S}$ represents the line projection in the mirror surface. The projection $\mathcal{S}$ of $\mathcal{L}$ in the image is then obtained using perspective or orthographic mapping. It can be shown (using (1) and (2) or following [3]) that 3D points lying on $\mathcal{L}$ are mapped into points in the image $\mathrm{x}$ which verify:

$$
\mathbf{x}^{T} \Omega \mathbf{x}=0
$$

with :

$$
\boldsymbol{\Omega}=\left(\begin{array}{ccc}
\alpha n_{x}^{2}-n_{z}^{\eta} \xi^{2} & \alpha n_{x} n_{y} & \beta n_{x} n_{z}^{\eta-1} \\
\alpha n_{x} n_{y} & \alpha n_{y}^{2}-n_{z}^{\eta} \xi^{2} & \beta n_{y} n_{z}^{\eta-1} \\
\beta n_{x} n_{z}^{\eta-1} & \beta n_{y} n_{z}^{\eta-1} & n_{z}^{\eta}
\end{array}\right)
$$

where $\alpha=1-\xi^{2}, \beta=2 \eta-3, \eta=2$ in the general case and $\eta=1$ for the combination parabolic mirrororthographic camera. A line in space is thus mapped onto the image plane to a conic curve. The relation (3) defines a quadratic equation:

$$
A_{0} x^{2}+A_{1} y^{2}+2 A_{2} x y+2 A_{3} x+2 A_{4} y+A_{5}=0
$$

with:

$$
\begin{array}{lll}
A_{0}=\alpha n_{x}^{2}-n_{z}^{\eta} \xi^{2} & A_{1}=\alpha n_{y}^{2}-n_{z}^{\eta} \xi^{2} & A_{2}=\alpha n_{x} n_{y} \\
A_{3}=\beta n_{x} n_{z}^{\eta-1} & A_{4}=\beta n_{y} n_{z}^{\eta-1} & A_{5}=n_{z}^{\eta}
\end{array}
$$

Let us note that the equation (4) is defined up to a scale factor. We thus normalize (4) using $A_{5}$ to obtain unambiguous representations, the quadratic equation is thus rewritten as follow:

$$
B_{0} x^{2}+B_{1} y^{2}+2 B_{2} x y+2 B_{3} x+2 B_{4} y+1=0
$$

with $B_{i}=\frac{A_{i}}{A_{5}}$. The case $n_{z}=0$ corresponds to a degenerate configuration of our representation where the optical 


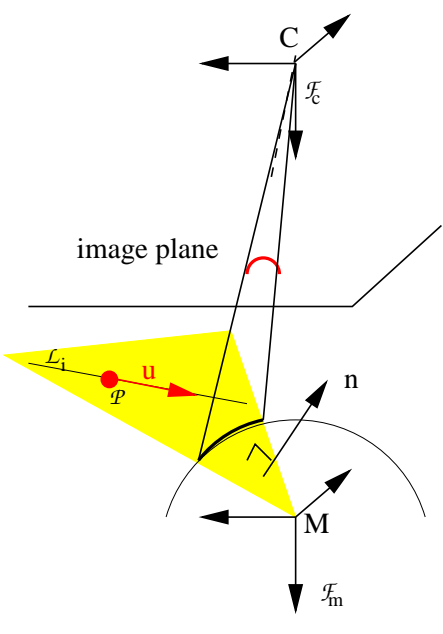

Fig. 2. Projection of line onto conic in the image plane

axis lies on the interpretation plane. In the following, we consider that $n_{z} \neq 0$. Let us note that the normal vector $\mathbf{n}$ can be computed from (5) since $\|\mathbf{n}\|=1$.

$$
\left\{\begin{array}{l}
n_{z}=\left(\frac{B_{3}^{2}+B_{4}^{2}}{\beta^{2}}+1\right)^{-1 / 2}=B_{n} \\
n_{x}=\frac{B_{3} B_{n}}{\beta} \\
n_{y}=\frac{B_{4} B_{n}}{\beta}
\end{array}\right.
$$

Since $\mathbf{n}^{\mathbf{T}} \mathbf{u}=0$, note also that $u_{z}$ can be rewritten as :

$$
u_{z}=-\frac{B_{3} u_{x}+B_{4} u_{y}}{\beta}=B_{u}
$$

\section{INTERACTION MATRIX OF CENTRAL CATADIOPTRIC CAMERAS FOR CONICS}

\section{A. Control law}

Consider the vector $\mathbf{s}=\left(\mathbf{s}_{\mathbf{1}}^{T}, \mathbf{s}_{\mathbf{2}}{ }^{T}, \cdots \mathbf{s}_{\mathbf{n}}{ }^{T}\right)^{T}$, where $\mathbf{s}_{\mathbf{i}}$ is a m-dimensional vector containing the visual observations used as input of the image-based control scheme. If the $3 \mathrm{D}$ features corresponding to visual observations are motionless, the time derivative of $\mathbf{s}_{\mathbf{i}}$ is: $\dot{\mathbf{s}_{\mathbf{i}}}=\frac{\partial \mathbf{s}_{i}}{\partial \mathbf{r}} \frac{d \mathbf{r}}{d t}=\mathbf{J}_{i} \mathbf{T}$ where $\mathbf{T}$ is a 6 -dimensional vector denoting the velocity of the central catadioptric camera and containing the instantaneous angular velocity $\boldsymbol{\omega}$ and the instantaneous linear velocity $\mathbf{v}$ of a given point expressed in the mirror frame. The $m \times 6$ matrix $\mathbf{J}_{i}$ is the interaction matrix (or image Jacobian). It links the variation of the visual observations to the camera velocity. If we consider the time derivative of $\mathbf{s}$, the corresponding interaction matrix is $\mathbf{J}=\left(\mathbf{J}_{i}, \cdots, \mathbf{J}_{n}\right)^{T}$ and $\dot{\mathbf{s}}=\mathbf{J T}$. To design an image-based control law, we use the task function approach introduced by Samson et al in [19]. Consider the task function $\mathbf{e}=\widehat{\mathbf{J}}^{+}\left(\mathbf{s}-\mathbf{s}^{*}\right)$ to be regulated to $\mathbf{0}\left(\mathbf{s}^{*}\right.$ is the desired value of the observation vector $\mathbf{s}$ and $\widehat{\mathbf{J}}^{+}$is the pseudo-inverse of a chosen model of J). A very simple control law can be designed by trying to ensure a decoupled exponential decay of the task function:

$$
\mathbf{T}=-\lambda \mathbf{e}=-\lambda \widehat{\mathbf{J}}^{+}\left(\mathbf{s}-\mathbf{s}^{*}\right)
$$

In order to compute the control law (9) it is necessary to provide an approximated interaction matrix $\widehat{\mathbf{J}}$. In the sequel, a generic analytical form of the interaction matrix for central catadioptric system is derived from the projection model of lines.

\section{B. Generic Image Jacobian}

Let us first define the observation vector $\mathbf{s}_{i}$ for a projected line (conic) in the central catadioptric image as:

$$
\mathbf{s}_{i}=\left[\begin{array}{lllll}
B_{0} & B_{1} & B_{2} & B_{3} & B_{4}
\end{array}\right]^{T}
$$

and the observation vector for $n$ conics as $\mathbf{s}=$ $\left(\mathbf{s}_{1}^{T} \cdots \mathbf{s}_{n}^{T}\right)^{T}$. As we will see in the sequel, the observation vector can be reduced for particular central catadioptric cameras such as the parabolic one. The interaction matrix for the observation vector $\mathbf{s}_{n}$ is:

$$
\mathbf{J}_{i}=\frac{\partial \mathbf{s}_{i}}{\partial \mathbf{r}}=\frac{\partial \mathbf{s}_{i}}{\partial \mathbf{n}_{i}} \frac{\partial \mathbf{n}_{i}}{\partial \mathbf{r}}=\mathbf{J}_{s n i} \mathbf{J}_{n i}
$$

where $\mathbf{n}_{i}=\left(n_{x i}, n_{y i}, n_{z i}\right)^{T}$ is the normal vector to the interpretation plane for line $\mathcal{L}_{i}$ expressed in the mirror frame, $\mathbf{J}_{s n i}$ represents the interaction between the visual observation motion and the normal vector variation, and $\mathbf{J}_{n i}$ links the normal variations to the camera motions. It can be shown that [1]:

$$
\left\{\begin{array}{l}
\dot{\mathbf{u}}_{i}=-\boldsymbol{\omega} \times \mathbf{u}_{i} \\
\dot{\mathbf{n}}_{i}=\frac{\partial \mathbf{n}_{i}}{\partial \mathbf{r}} \mathbf{T}=\frac{\mathbf{v}^{T} \mathbf{n}_{i}}{h}\left(\mathbf{u}_{i} \times \mathbf{n}_{i}\right)-\boldsymbol{\omega} \times \mathbf{n}_{i}
\end{array}\right.
$$

where $h=\|\overrightarrow{M \mathcal{P}} \times \mathbf{u}\|$. According to the previous equation, the interaction between the normal vector and the sensor motion is thus:

$$
\mathbf{J}_{n i}=\left(\begin{array}{cc}
\frac{1}{h}\left(\mathbf{u}_{i} \times \mathbf{n}_{i}\right) \mathbf{n}_{i}^{T} & \left.\left[\mathbf{n}_{i}\right]_{\times}\right)
\end{array}\right.
$$

where $[\mathbf{n}]_{\times}$denotes the antisymetric matrix associated to the vector $\mathbf{n}$. The Jacobian $\mathbf{J}_{s n i}$ is obtained by computing the partial derivative of (10) with respect to $\mathbf{n}_{i}$ and using (7):

$$
\mathbf{J}_{s n i}=\frac{1}{\beta B_{n}^{\eta}}\left(\begin{array}{ccc}
2 \alpha B_{3} B_{n} & 0 & -\frac{\eta \alpha}{\beta} B_{3}^{2} B_{n} \\
0 & 2 \alpha B_{4} B_{n} & -\frac{\eta \alpha}{\beta} B_{4}^{2} B_{n} \\
\alpha B_{4} B_{n} & \alpha B_{3} B_{n} & -\frac{\eta \alpha}{\beta} B_{3} B_{4} B_{n} \\
\beta^{2} B_{n}^{\eta-1} & 0 & -\beta B_{3} B_{n}^{\eta-1} \\
0 & \beta^{2} B_{n}^{\eta-1} & -\beta B_{4} B_{n}^{\eta-1}
\end{array}\right)
$$

The interaction matrix can finally be computed by combining the equations (12) and (13) according to relation (11). Note that the rank of the interaction matrix given by (11) is 2 . At least three lines are thus necessary to control the 6 dof of a robotic arm. As can be seen on equation (12), only the $3 \mathrm{D}$ parameters $u_{x}$ and $u_{y}$ have to be introduced in the interaction matrix. As usual when visual informations are used in image-based control, these parameters only act on the translation velocities. As previously explained, a chosen estimation of the interaction matrix is used to design the control law. The value of $\mathbf{J}$ at the desired position is a typical choice. In this case, the 3D parameters have to be estimated only for the desired position. In the next part, we study the particular case of paracatadioptric camera (parabolic mirror combined to orthographic camera). 


\section{A case study: paracatadioptric cameras}

In the case of paracatadioptric cameras, we have $\xi=1$, $\alpha=0$ and $\eta=1$. The lines are projected onto the image plane as circles. It can be noticed that $A_{2}=0$ and $A_{0}=A_{1}=-A_{5}$ and thus the observation vector can be reduced as $\mathbf{s}_{\mathbf{i}}=\left[\begin{array}{ll}B_{3} & B_{4}\end{array}\right]^{T}$. Note also that a line is projected as a circle of center $x_{c}=B_{3}, y_{c}=B_{4}$ and radius $\left(B_{3}^{2}+B_{4}^{2}+1\right)^{1 / 2}$. Minimizing the task function e can thus be interpreted as minimizing the distance between current and desired centers of circles by moving the camera. According to equation (13), the Jacobian $\mathbf{J}_{\text {sni }}$ can be reduced as follow:

$$
\mathbf{J}_{s n i}=-\frac{1}{B_{n}}\left(\begin{array}{ccc}
1 & 0 & B_{3} \\
0 & 1 & B_{4}
\end{array}\right)
$$

The rank of the image Jacobian is 2. The six degrees of freedom of a robotic arm can thus be fully controlled using three projected lines as long as the lines define three different interpretations planes.

\section{Simulation RESUlts}

In this section, we present simulation results of central catadioptric visual servoing from lines. In the first simulations, we have considered two positioning tasks of a 6 dof robotic arm. The last simulation concerns a task of line following with a mobile robot.

\section{A. Positioning task}

The value of $\mathbf{J}$ at the desired position has been used. We have considered two positioning tasks. From an initial position, the robot has to reach a desired position expressed as a desired observation vector. The first simulation concerns a camera combining an hyperbolic mirror and a perspective camera (Figs. 4, 5 and 6). The second simulation concerns a camera combining a parabolic mirror and an orthographic camera (Figs. 7, 8 and 9). The initial attitude of the camera with respect to the world frame is given by $\mathbf{r}_{i}=$ $[0,0,1,0,0,0]^{T}$ (the first three components are the translations in meter and the last three are the rotations in radian). The desired image corresponds to the camera configuration given by $\mathbf{r}_{d}=\left[0.1,0.1,1.1, \frac{\pi}{8}, \frac{\pi}{8}, \frac{\pi}{8}\right]^{T}$ in the world frame. The three lines are defined in the world space by the following Plücker coordinates:

$$
\left\{\begin{array}{c}
\mathcal{L}_{1}:\left(\begin{array}{c}
u_{1}=\left(\begin{array}{lll}
0 & 1 & 0
\end{array}\right)^{T} \\
n_{1}=\left(\begin{array}{lll}
0 & 0 & -1
\end{array}\right)^{T}
\end{array}\right) \\
\mathcal{L}_{2}:\left(\begin{array}{c}
u_{2}=\left(\begin{array}{lll}
0 & 0.9806 & 0.1961
\end{array}\right)^{T} \\
n_{2}=\left(\begin{array}{lll}
0 & -0.1961 & 0.9806
\end{array}\right)^{T}
\end{array}\right) \\
\mathcal{L}_{3}:\left(\begin{array}{c}
u_{3}=\left(\begin{array}{lll}
0.9623 & 0.1925 & 0.1925
\end{array}\right)^{T} \\
n_{3}=\left(\begin{array}{lll}
0.1961 & 0 & -0.9806
\end{array}\right)^{T}
\end{array}\right)
\end{array}\right.
$$

Figure 3 shows the initial spatial configurations of lines and camera. Image noise has been introduced (additive noise with maximum amplitude of 1 pixel) in the observation vectors. The Plücker coordinates of the considered lines with respect to the world frame have been corrupted with errors of maximal amplitude of $5 \%$ (these errors corrupt

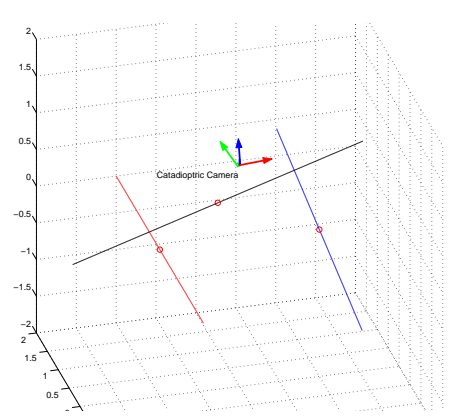

Fig. 3. Lines confi gurations in $3 \mathrm{D}$

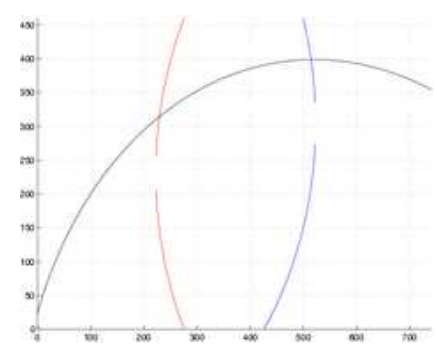

(a)

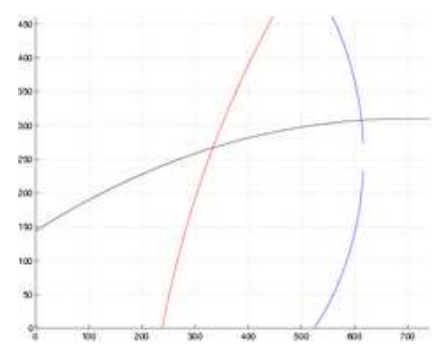

(b)

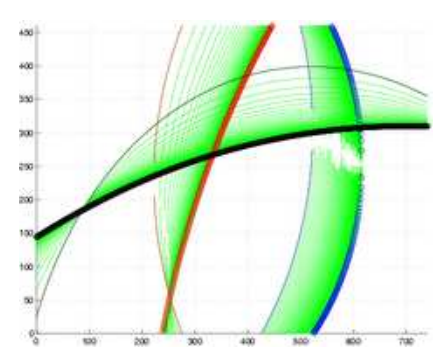

(c)

Fig. 4. Hyperbolic mirror-perspective camera: (a) Initial image, (b) desired image, (c) trajectories in the image plane of line projection

the estimation of the interaction matrix at the desired configuration). The images corresponding to the initial and desired cameras positions are given in Figures 4(a) and 4(b) for the hyperbolic-perspective camera 7(a) and 7(b) for the parabolic-orthographic camera. Figures 4(c) and 7(c) shows the trajectories of the conics in the image plane. Camera velocities are given in Figures 5 and 8. As can been seen in Figures 6 and 9 showing the errors between desired and current observation vectors, the positioning task is correctly realized as well for the hyperbolic-perspective camera as for the parabolic-orthographic camera. Note finally, that these results confirm that visual servoing schemes can benefit from the use of central catadioptric vision systems to cope with visibility constraints.

\section{B. Line following}

Catadioptric image-based visual servoing from lines can be used to achieve trajectory following with a mobile robot. In this simulation, the angular deviation of a mobile robot with respect to a straight line is regulated to a desired value using its projection in the paracatadioptric image (parabolic mirror and orthographic projection). In this case, only one 


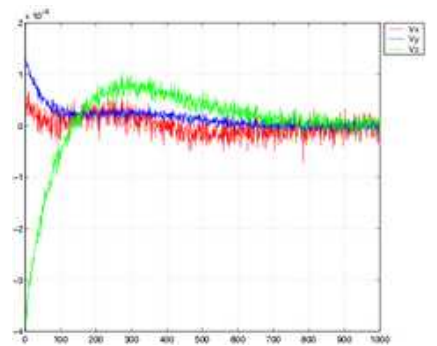

(a)

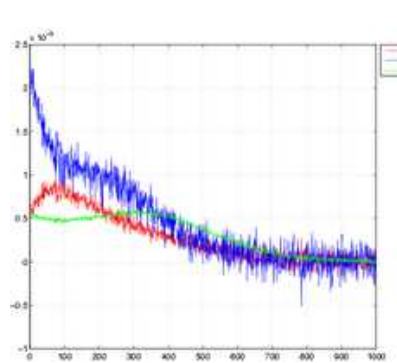

(b)
Fig. 5. Hyperbolic mirror-perspective camera: (a) Translational velocities $[\mathrm{m} / \mathrm{s}]$ and (b) rotational velocities $[\mathrm{rad} / \mathrm{s}]$

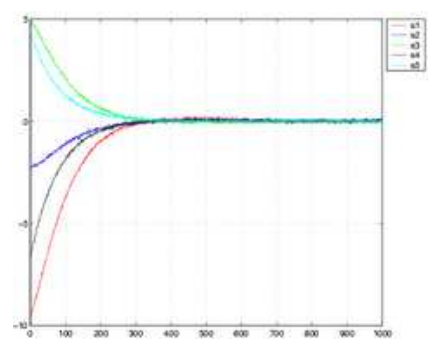

(a)

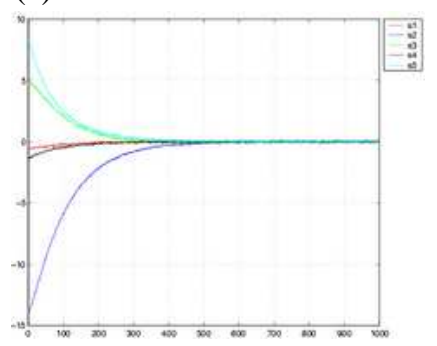

(c)

Fig. 6. Hyperbolic mirror-perspective camera $\left(\mathbf{s}-\mathbf{s}^{*}\right)$ : (a) Errors for the fi rst conic, (b) Errors for the second conic, (c) Errors for the third conic

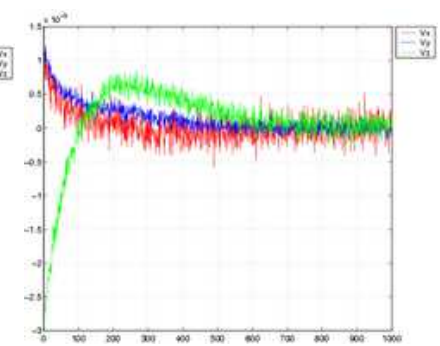

(a)

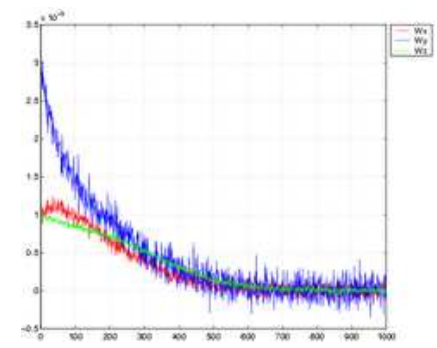

(b)
Fig. 8. Parabolic mirror-orthographic camera: (a) Translational velocities $[\mathrm{m} / \mathrm{s}]$ and $(\mathrm{b})$ rotational velocities $[\mathrm{rad} / \mathrm{s}]$

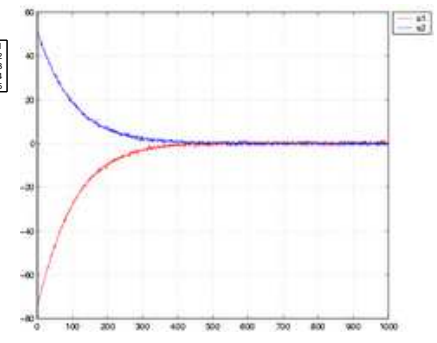

(a)

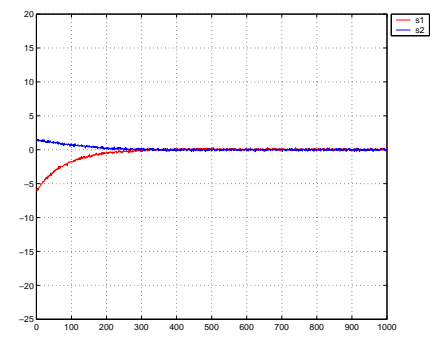

(b)

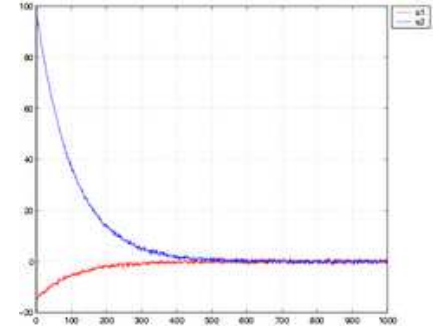

(c)
Fig. 9. Parabolic mirror-orthographic camera $\left(\mathbf{s}-\mathbf{s}^{*}\right)$ : (a) Errors for the fi rst conic, (b) Errors for the second conic, (c) Errors for the third conic

degree of freedom has to be controlled and thus one feature extracted from the image is necessary. The camera frame is supposed confounded with the robot control frame. In this configuration, the camera frame and the mobile robot are thus subjected to the same kinematic constraints. The kinematic screw is only composed with a linear velocity along the $x$-axis and an angular velocity about the optical $\rightarrow$ axis. To drive the mobile robot parallel with respect to a 3D line (see Fig. 10(d)), the linear velocity can be taken as a constant and only the lateral deviation (the rotation about the $z$-axis) has to be controlled. As already explained, a line is projected as a circle of center $x_{c}=B_{3}, y_{c}=B_{4}$ and radius $r=\left(B_{3}^{2}+B_{4}^{2}+1\right)^{1 / 2}$. It is clear that the radius does not vary if the camera is rotated with respect to the $z$-axis of the camera frame. If $s=B_{3}+B_{4}$ is chosen has visual observation, the interaction is given by $J_{i}=\frac{\partial s}{\partial \alpha_{z}}=-B_{4}+B_{3}$ and the control law (9) can be used.

The images corresponding to the initial and desired cameras positions are given in Figures 10(a) and 10(b). Figures 10(c) shows the trajectories of the circle in the image plane. Angular velocity is given in Figure 11(b). As

Fig. 7. Parabolic mirror-orthographic camera: (a) Initial image , (b) desired image (c) trajectory of the catadioptric image lines 


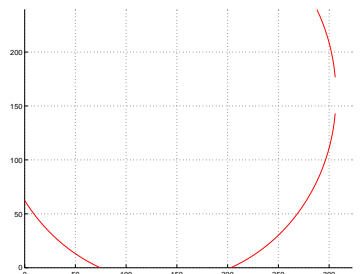

(a)

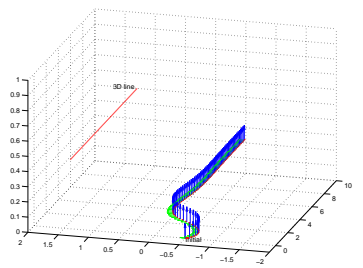

(c)

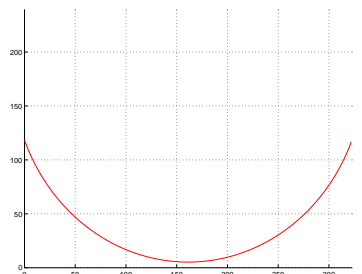

(b)

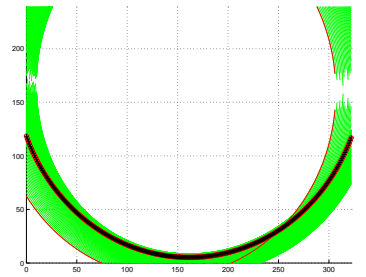

(d)
Fig. 10. (a) Initial image, (b) desired image, (c) trajectories in the image plane of line projection (d) robot trajectory

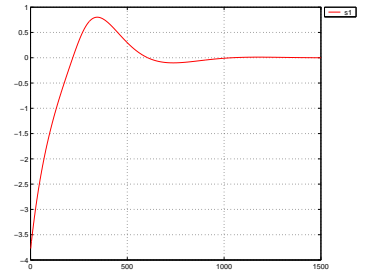

(a)

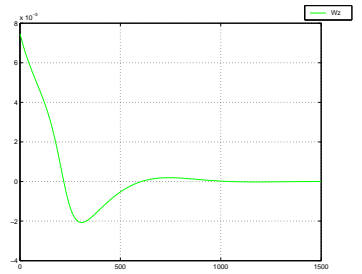

(b)
Fig. 11. (a) Error in the image $s-s^{*}$, (b) rotational velocities [rad/s]

can been seen in Figure 11(a) showing the errors between desired and current observation vectors the task is correctly realized.

\section{CONCLusions}

Visibility constraints are extremely important for visual servoing applications. To overcome these constraints, the wide field of view of central catadioptric cameras can be exploited. We have addressed the problem of controlling a robotic system by incorporating observations from a central catadioptric camera. A generic image Jacobian which can be used to design image-based control laws, has been derived from the model of line projection. Future work will be devoted to integrate in the control law nonholonomic constraints and to study path planning in central catadioptric image space.

\section{ACKNOWLEDGMENT}

This work has been funded by the project OMNIBOT of ROBEA:'Robotique mobile et Entits Artificielles".

\section{REFERENCES}

[1] N. Andreff, B. Espiau, and R. Horaud. Visual servoing from lines. Int. Journal of Robotics Research, 21(8):679-700, August 2002

[2] S. Baker and S. K. Nayar. A theory of single-viewpoint catadioptric image formation. Int. Journal of Computer Vision, 35(2):1-22, Nov. 1999.

[3] J. Barreto and H. Araujo. Geometric properties of central catadioptric line images. In 7th European Conf. on Computer Vision, ECCV'02, pages 237-251, Copenhagen, Denmark, May 2002.
[4] J. P. Barreto, F. Martin, and R. Horaud. Visual servoing/tracking using central catadioptric images. In 8th Int. Symp. on Experimental Robotics, ISER'02, pages 863-869, Bombay, India, July 2002.

[5] R. Benosman and S. Kang. Panoramic Vision. Springer Verlag, 2000.

[6] P. Blaer and P.K. Allen. Topological mobile robot localization using fast vision techniques. In IEEE Int. Conf. on Robotics and Automation, ICRA'02, pages 1031-1036, Washington, USA, May 2002.

[7] D. Burshka, J. Geiman, and G. Hager. Optimal landmark confi guration for vision based control of mobile robot. In IEEE Int. Conf. on Robotics and Automation, ICRA'03, pages 3917-3922, Tapei, Taiwan, Sept. 2003.

[8] F. Chaumette. Potential problems of stability and convergence in image-based and position-based visual servoing. The Confluence of Vision and Control, LNCIS Series, Springer Verlag, 237:66-78, 1998.

[9] G. Chesi, K. Hashimoto, D. Prattichizzo, and A. Vicino. A switching control law for keeping features in the fi eld of view in eye-in-hand visual servoing. In IEEE Int. Conf. on Robotics and Automation, ICRA'03, pages 3929-3934, Taipei, Taiwan, September 2003.

[10] N. J. Cowan, J. D. Weingarten, and D. E. Koditschek. Visual servoing via navigation functions. IEEE Trans. on Robotics and Automation, 18(4):521-533, August 2002.

[11] B. Espiau, F. Chaumette, and P. Rives. A new approach to visual servoing in robotics. IEEE Trans. on Robotics and Automation, 8(3):313-326, June 1992.

[12] C. Geyer and K. Daniilidis. A unifying theory for central panoramic systems and practical implications. In European Conf. on Computer Vision, ECCV'O0, volume 29, pages 159-179, Dublin, Ireland, May 2000.

[13] S. Hutchinson, G.D. Hager, and P.I. Corke. A tutorial on visual servo control. IEEE Trans. on Robotics and Automation, 12(5):651-670, October 1996.

[14] E. Malis, J. Borrelly, and P. Rives. Intrinsics-free visual servoing with respect to straight lines. In IEEE/RSJ Int. Conf. on Intelligent Robots Systems, IROS'02, Lausanne, Switzerland, October 2002.

[15] E. Malis, F. Chaumette, and S. Boudet. $21 / 2$ d visual servoing. IEEE Trans. on Robotics and Automation, 15(2):238-250, April 1999.

[16] Y. Mezouar and F. Chaumette. Path planning for robust image-based control. IEEE Trans. on Robotics and Automation, 18(4):534-549, August 2002.

[17] A. Paulino and H. Araujo. Multiple robots in geometric formation: Control structure and sensing. In Int. Symp. on Intelligent Robotic Systems, pages 103-112, Reading, UK, July 2000.

[18] E. Malis S. Benhimane. Vision-based control with respect to planar and non-planar objects using a zooming camera. In IEEE Int. Conf. on Advanced Robotics, ICAR'04, pages 863-869, July 2003.

[19] C. Samson, B. Espiau, and M. Le Borgne. Robot Control : The Task Function Approach. Oxford University Press, 1991.

[20] B. Thuilot, P. Martinet, L. Cordesses, and J. Gallice. Position-based visual servoing: keeping the object in the fi eld of vision. In IEEE Int. Conference on Robotics and Automation, ICRA'02, pages 16241629, Washington DC, USA, May 2002.

[21] R. Vidal, O. Shakernia, and S. Sastry. Formation control of nonholonomic mobile robots with omnidirectional visual servoing and motion segmentation. In IEEE Int. Conf. on Robotics and Automation, ICRA'03, Taipei, Taiwan, September 2003.

[22] N. Winter, J. Gaspar, G. Lacey, and J. Santos-Victor. Omnidirectional vision for robot navigation. In IEEE Workshop on Omnidirectional Vision, OMNIVIS'00, pages 21-28, South Carolina, USA, June 2000. 